\title{
SHORT REPORT \\ Group A streptococcal strains isolated in Lao People's Democratic Republic from 2004 to 2013
}

\author{
S. RATTANAVONG ${ }^{1}$, D. A. B. DANCE ${ }^{1,2}$, V. DAVONG ${ }^{1}$, C. BAKER ${ }^{3}$, \\ H. FROST ${ }^{3}$, R. PHETSOUVANH ${ }^{1}$, M. VONGSOUVATH ${ }^{1}$, P. N. NEWTON ${ }^{1,2}$, \\ A. C. STEER ${ }^{3,4}$ AND P. R. SMEESTERS $S^{3,4,5 *}$ \\ ${ }^{1}$ Lao-Oxford-Mahosot Hospital Wellcome Trust Research Unit, Microbiology Laboratory, Mahosot Hospital, \\ Vientiane, Lao People's Democratic Republic \\ ${ }^{2}$ Centre for Tropical Medicine and Global Health, Old Road Campus, University of Oxford, Oxford, UK \\ ${ }^{3}$ Group A Streptococcus Research Group, Murdoch Children's Research Institute, Melbourne, Australia \\ ${ }^{4}$ Centre for International Child Health, University of Melbourne, Melbourne, Australia \\ ${ }^{5}$ Department of Paediatrics, Hôpital Universitaire des Enfants Reine Fabiola, Brussels, Belgium
}

Received 7 September 2015; Final revision 29 October 2015; Accepted 29 October 2015; first published online 8 December 2015

\section{SUMMARY}

Epidemiological data regarding group A streptococcal (GAS) infections in South East Asia are scarce with no information from Laos. We characterized emm types, emm clusters and the antibiotic resistance profile of 124 GAS isolates recovered in Laos during 2004-2013. Most strains were recovered from skin and invasive infections ( $76 \%$ and 19\%, respectively). Thirty-four emm types were identified as belonging to $12 \mathrm{emm}$ clusters and no novel emm types were identified. No significant differences were observed in the distribution of emm types or emm clusters according to age or site of recovery (skin or invasive infections). There was moderate strain diversity in this country but considerable differences in emm-type distribution between Laos, Thailand and Cambodia. Vaccine coverage was high for the $\mathrm{J} 8$ vaccine candidate. The theoretical coverage for the 30-valent vaccine candidate needs further investigation. Antibiotic resistance was moderate to erythromycin and chloramphenicol ( $8 \%$ and $7 \%$, respectively) and low to ofloxacin $(<1 \%)$.

Key words: Molecular epidemiology, Streptococcus pyogenes, vaccines.

Group A streptococci (Streptococcus pyogenes; GAS) cause significant morbidity and mortality globally with most of the disease burden occurring in lowand middle-income settings. With no effective control strategies available, a GAS vaccine is urgently needed. The most advanced vaccine candidates use the surface $\mathrm{M}$ protein as antigen. Two vaccine candidates have entered phase 1 clinical trials over the past decade with a further two vaccines planned for trials in

\footnotetext{
* Author for correspondence: Dr P. R. Smeesters, Group A Streptococcal Research Group, Murdoch Children's Research Institute, Flemington Road, Parkville, VIC 3052, Australia. (Email: pierre.smeesters@mcri.edu.au)
}

2015 [1]. The 30-valent type-specific vaccine candidate includes peptides from a selection of $\mathrm{M}$ proteins associated with disease burden in both high- and lowincome settings but questions have been raised regarding coverage in low-income settings where a high diversity of circulating emm types has been observed $[2,3]$. The $\mathbf{J} 8$ vaccine is based on a conserved region of the M protein (J8) and aims to provide broad protection across many strains. However, no fewer than 68 allelic variants have been described for $\mathrm{J} 8$ and the relationship between allelic diversity and vaccine efficacy has not yet been systematically characterized [1]. Importantly, limited epidemiological data regarding circulating emm types and $\mathbf{J} 8$ variants are available

This is an Open Access article, distributed under the terms of the Creative Commons Attribution licence (http://creativecommons.org/licenses/by/4.0/), which permits unrestricted re-use, distribution, and reproduction in any medium, provided the original work is properly cited. 
from a number of key regions of the world making vaccine-coverage estimates imprecise, especially from South East Asia with only one study originating from a low-income country (Cambodia) [2-4].

The Lao People's Democratic Republic (Laos) is a low- to middle-income country with a population of $\sim 6.9$ million people which, despite rapid economic growth, is still one of the poorest in South East Asia (http://data.worldbank.org/country/lao-pdr). It also has some of the worst health indicators in the region, with an average life expectancy of 65 years for males and 68 years for females, and an infant mortality rate of 54/1000 live births in 2013 (http://apps.who. int/gho/data/view.main.CM1320R?lang=en). In addition, very few diagnostic laboratories undertake bacterial culture, therefore data on the epidemiology and antimicrobial susceptibility of bacterial pathogens within Laos are very scarce.

The Microbiology Laboratory of Mahosot Hospital, the largest hospital in the capital, Vientiane, has conducted culture-based diagnosis of bacterial infections and storage of significant pathogens since 2000. We analysed these databases to identify GAS isolates recovered during a 10-year period (2004-2013). We extracted clinical details and demographic information of patients with GAS infection, including geographical coordinates of the addresses of the patients. Invasive disease was defined as the isolation of GAS from blood in a patient with a clinical infection. Skin infection, acute otitis media, and pharyngitis was defined by the presence of clinical symptoms associated with the isolation of GAS from the relevant site.

Identification of GAS was based on colony morphology, $\beta$-haemolysis on $5 \%$ goat blood agar, negative catalase reaction, and detection of Lancefield Group A antigen by latex agglutination (Streptococcal Grouping kit, Oxoid, UK). Antibiotic susceptibility to four antibiotics (penicillin, erythromycin, ofloxacin, chloramphenicol) was determined by the CLSI disk diffusion method and isolates were frozen at $-80^{\circ} \mathrm{C}$ prior to shipment to Melbourne for further testing. The isolates were re-confirmed as GAS as above and were then emm-typed according to the US Centers for Disease Control and Prevention protocol with minor modifications; primers MF2 and MR1 were used when primers 1 and 2 were not successful, as described previously [5]. In addition to emm-typing, we also classified isolates into $\mathrm{emm}$ clusters. This typing system classifies the many GAS emm types into 48 discrete emm clusters containing closely related M proteins that share binding and structural properties
Table 1. emm clusters and emm types of the GAS isolates characterized in the study

\begin{tabular}{lll}
\hline \hline emm cluster & Isolates $(n)$ & emm types $(n)$ \\
\hline E3 & 47 & $25(6), 44(28), 49(2), 58(4)$, \\
& & $79(3), 82(4)$ \\
E4 & 21 & $(1), 22(5), 73(2), 77(2), 89$ \\
& & $762(1), 109(8), 114(1)$ \\
E2 & 15 & $11(1), 63(2), 75(6), 81(3)$ \\
E6 & 12 & $33(2), 70(1), 101(1), 230(4)$ \\
D4 & 8 & $95(4)$ \\
M95 & 4 & Non-typable (4) \\
Non-typable & 4 & $4(1), 165(2)$ \\
E1 & 3 & $74(3)$ \\
M74 & 3 & $105(3)$ \\
M105 & 3 & $185(2)$ \\
M185 & 2 & $1(1)$ \\
A-C3 & 1 & $100(1)$ \\
D2 & 1 & \\
\hline \hline
\end{tabular}

[6-8]. emm clusters can be directly deduced by the emm-typing result and predict the $\mathrm{J} 8$ vaccine antigen content [6]. Coverage by the 30 -valent vaccine was estimated using the latest cross-opsonization data $[6,9,10]$. We used Simpson's Reciprocal Index (SRI) to assess strain diversity [3]. Regional molecular epidemiology was assessed by comparison of the data with a similar dataset from Thailand [11] and Cambodia [4].

The Oxford Tropical Research Ethics Committee and National Ethics Committee for Health Research, Government of Laos approved this study.

We characterized 124 GAS isolates. Median patient age was 35 years (interquartile range 7-56, range 0 92; data available for $121 / 124$ patients) and $31 \cdot 4 \%$ of the patients were aged $<15$ years at presentation. Most of the patients were diagnosed with a skin infection $(94 / 124,76 \%)$ followed by invasive infection (24/ $124,19 \%)$. There was no clinical information available for four patients. We identified $34 \mathrm{emm}$ types as belonging to $12 \mathrm{emm}$ clusters and no novel $\mathrm{emm}$ types were identified (Table 1). No significant differences were observed in the distribution of emm types or emm clusters according to age or site of recovery (skin or invasive infections). Four isolates were nontypable. While there were 34 different emm types observed among 124 GAS isolates in this study, the strain diversity as measured by the SRI (that takes into account both richness and evenness), was $13 \cdot 2$ [95\% confidence interval (CI) 9.6-21·5]. This indicates a relatively moderate strain diversity in comparison to data from children in Cambodia $(\mathrm{SRI}=28 \cdot 5)$ and 
(a)

Lao PDR

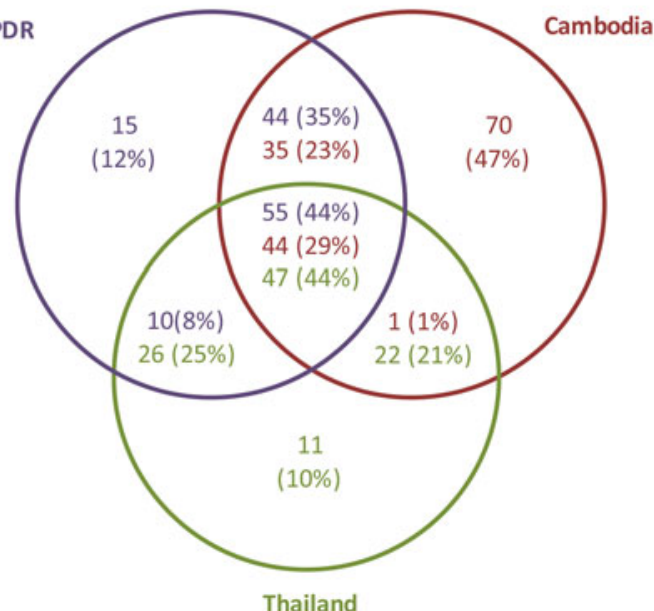

(b)
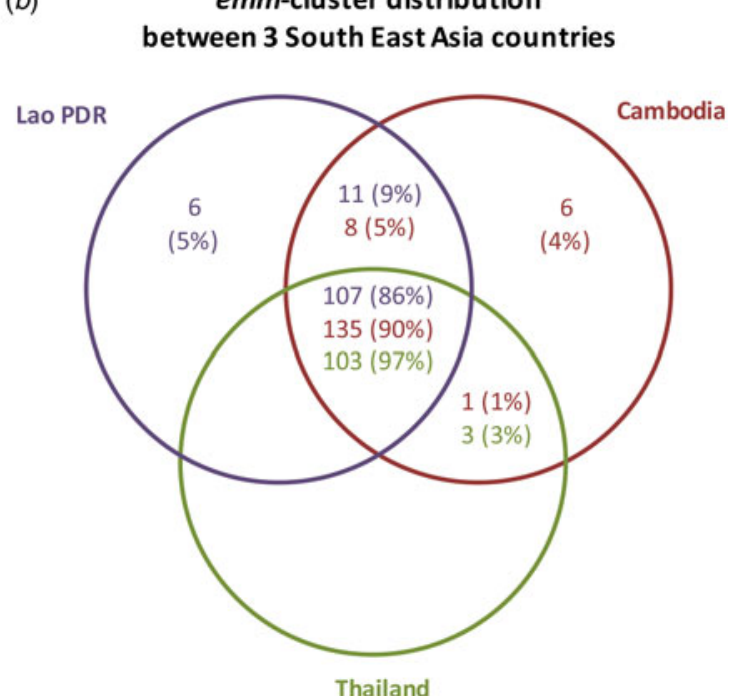

Fig. 1. Comparison of the GAS isolates recovered from 3 South East Asian countries. Distribution of emm types and emm clusters among GAS infections recovered from three Southeast Asian countries. The number of isolates (\%) associated with infections and belonging to specific and common (a) emm types and (b) emm clusters is shown for each country.

other other low-income countries [2, 4]. Geographical coordinates of the patient's home were available for $117 / 124$ patients included in this study and showed that $96 \%(112 / 117)$ of patients' homes were within $\sim 100 \mathrm{~km}$ radius of Vientiane, the main catchment area of Mahosot Hospital. This relatively small geographical area may partially account for the relatively low diversity of strains observed.

No isolate was resistant to penicillin; however, one isolate was resistant to ofloxacin (another was intermediate), nine ( $7 \%$ ) were resistant to chloramphenicol (three intermediate) and $10(8 \%)$ were resistant to erythromycin. These chloramphenicol- and erythromycin-resistant isolates belonged to seven and six different emm types, respectively. Of note, seven of the 10 erythromycin-resistant isolates were also resistant to chloramphenicol. These data represent the first information about GAS antibiotic resistance in South East Asia. Data from East Asia indicate rates of erythromycin resistance ranging from $8 \%$ to $14 \cdot 6 \%$ in Taiwan during the period 2010-2011 [12, 13], reaching as high as $>95 \%$ in China [14].

Sixty-one $(49 \%)$ isolates belonged to emm types included in the 30 -valent vaccine candidate. Thirtyeight $(31 \%)$ isolates were emm types that have been shown to be cross-opsonized in a rabbit model and therefore have the potential to be covered by the 30 -valent vaccine. Taking into account this crossopsonization effect, the theoretical coverage could be
$80 \%$ (95\% CI 73-87), but may be even higher since $19 \%$ of the isolates belonged to eight emm types that had not yet been tested for cross-opsonization. These eight $\mathrm{emm}$ types should be investigated for potential cross-opsonization in order to better assess the theoretical coverage of the 30 -valent vaccine in Laos. In terms of theoretical coverage of the J8 vaccine candidate, $19(15 \%)$ and $101(82 \%)$ of the isolates were predicted to contain the $\mathrm{J} 8$ and $\mathrm{J} 8 \cdot 1$ allele, respectively. Therefore, J 8 vaccine coverage could be expected to be $97 \%$ (95\% CI $94-100)$.

We compared these data to previous data from Thailand and Cambodia. The Thailand dataset comprises 106 GAS isolates from skin and throat collected between 1985 and 2004, including those from patients with rheumatic heart disease [11]. The Cambodian dataset comprises 150 GAS isolates recovered from skin, throat and invasive infections in children collected between 2007 and 2012 [4]. The emm-type distribution between these three countries shows that nearly half of the Cambodian isolates belong to an emm type which has not been recovered in the two other countries (Fig. 1). By contrast, only 10 and $12 \%$ of the strains isolated in Thailand and Laos, respectively, belonged to an emm type not recovered in the other two countries. The emm cluster distribution shows that $86-97 \%$ of the strains belong to an emm cluster present in all three countries 
(Fig. 1). Moreover, the distribution also shows that the apparently distinct epidemiology of GAS in Cambodia does not correlate with the presence of specific emm clusters.

The most important limitation of our study is its retrospective nature. In future, prospective and comprehensive GAS infection surveillance in Laos will be highly desirable. Other limitations of our study are the relative absence of pharyngeal isolates from the collection and the restricted geographical sampling area. Finally, caution should be exercised when comparing the Lao, Thai and Cambodian datasets given the methodological differences in sampling and study periods. Notably, a potential confounding factor for the differences in strain distribution is that the Cambodian isolates were all from children. Better prospective data obtained using similar methodologies in terms of study period, inclusion criteria and targeted population should be obtained before firm conclusions can be drawn.

Overall, these data indicate a relatively low diversity of circulating GAS strains in the central region from Laos, a high coverage of the $\mathrm{J} 8$ vaccine candidate and the need for complementary studies to assess the potential coverage of the 30 -valent vaccine candidate. These results also confirm the importance of epidemiological studies at a local and regional level to inform vaccine development.

\section{ACKNOWLEDGEMENTS}

We thank all the doctors and nursing staff of Mahosot Hospital, the staff of the microbiology laboratory, the Directors of Mahosot Hospital, the Minister of Health and the Director of the Curative Department, Ministry of Health, for their support of this study.

This research was supported by the Wellcome Trust, and an internal grant from the Murdoch Children's Research Institute.

\section{DECLARATION OF INTEREST}

None.

\section{REFERENCES}

1. Moreland NJ, et al. Working towards a group A streptococcal vaccine: report of a collaborative Trans-Tasman workshop. Vaccine 2014; 32: 3713-3720.

2. Steer AC, et al. Global emm type distribution of group A streptococci: systematic review and implications for vaccine development. Lancet Infectious Diseases 2009; 9: 611-616.

3. Smeesters PR, et al. Differences among group A streptococcus epidemiological landscapes: consequences for M protein-based vaccines? Expert Reviews Vaccines 2009; 8: 1705-1720.

4. Turner PN, et al. Molecular epidemiology of Group A Streptococcus infections in Cambodian children, 20072012. Pediatric Infectious Disease Journal (in press).

5. Smeesters PR, et al. Differences between Belgian and Brazilian group A Streptococcus epidemiologic landscape. PLoS ONE 2006; 1: e10.

6. Sanderson-Smith M, et al. A systematic and functional classification of Streptococcus pyogenes that serves as a new tool for molecular typing and vaccine development. Journal of Infectious Diseases 2014; 210: 1325-1338.

7. Baroux N, et al. The emm-cluster typing system for Group A Streptococcus identifies epidemiologic similarities across the Pacific Region. Clinical Infectious Diseases 2014; 59: e84-92.

8. Shulman ST, et al. Added value of the emm-cluster typing system to analyze Group A Streptococcus epidemiology in high-income settings. Clinical Infectious Diseases 2014; 59: 1651-1652.

9. Dale JB, et al. New 30-valent M protein-based vaccine evokes cross-opsonic antibodies against non-vaccine serotypes of group A streptococci. Vaccine 2011; 29: 81758178.

10. Dale JB, et al. Potential coverage of a multivalent $M$ protein-based group A streptococcal vaccine. Vaccine 2013; 31: 1576-81.

11. Yoonim $\mathbf{N}$, et al. $\mathrm{M}$ protein typing of Thai group A streptococcal isolates by PCR-Restriction fragment length polymorphism analysis. BMC Microbiol 2005; 5: 63.

12. Wu PC, et al. Molecular characterization of group A streptococcal isolates causing scarlet fever and pharyngitis among young children: a retrospective study from a northern Taiwan medical center. Journal of Microbioliology, Immunology, and Infectious Diseases 2014; 47: 304-310.

13. Huang CY, et al. Epidemiology and molecular characterization of macrolide-resistant Streptococcus pyogenes in Taiwan. Journal of Clinical Microbiology 2014; 52: 508-516.

14. Zhou W, et al. Erythromycin-resistant genes in group A beta-haemolytic Streptococci in Chengdu, Southwestern China. Indian Journal of Medical Microbiology 2014; 32: 290-293. 